\title{
CDISC Multiple Sclerosis Therapeutic Area User Guide Version 1.0
}

National Cancer Institute

\section{Source}

National Cancer Institute. CDISC Multiple Sclerosis Therapeutic Area User Guide Version

1.0. NCl Thesaurus. Code C161467.

The 1.0 version of the CDISC Multiple Sclerosis therapeutic area user guide. 